De l'« illusionnisme monumental » au monument illusionniste : réflexions sur les usages de la microarchitecture au début du $\mathrm{XV}^{\mathrm{e}}$ siècle

Jean-Marie Guillouët

\title{
OpenEdition
}

1 Journals

Édition électronique

URL : https://journals.openedition.org/cem/17108

DOI : $10.4000 /$ cem. 17108

ISSN : 1954-3093

Éditeur

Centre d'études médiévales Saint-Germain d'Auxerre

Référence électronique

Jean-Marie Guillouët, « De l'« illusionnisme monumental » au monument illusionniste : réflexions sur les usages de la microarchitecture au début du xv siècle », Bulletin du centre d'études médiévales d'Auxerre | BUCEMA [En ligne], 24.1 | 2020, mis en ligne le 21 septembre 2020, consulté le 22 septembre 2022. URL : http://journals.openedition.org/cem/17108; DOI : https://doi.org/10.4000/ cem. 17108

Ce document a été généré automatiquement le 22 septembre 2022.

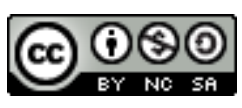

Creative Commons - Attribution - Pas d'Utilisation Commerciale - Partage dans les Mêmes Conditions 4.0 International - CC BY-NC-SA 4.0

https://creativecommons.org/licenses/by-nc-sa/4.0/ 


\section{De l'« illusionnisme monumental » au monument illusionniste : réflexions sur les usages de la microarchitecture au début du $\mathrm{XV}^{\mathrm{e}}$ siècle}

Jean-Marie Guillouët

1 À l'occasion d'un article paru en 1999 dans les mélanges en l'honneur d'Anne Prache, Fabienne Joubert avait proposé d'employer l'expression «illusionnisme monumental » à propos de deux ensembles célèbres situés à l'ourlet des $\mathrm{XIV}^{\mathrm{e}}$ et $\mathrm{XV}^{\mathrm{e}}$ siècles : les vitraux de la SainteChapelle de Bourges et le tombeau de Philippe le Hardi de la chartreuse de Champmol (fig. 1) ${ }^{1}$.

2 L'expression servait à désigner les expériences conduites concurremment dans ces œuvres par André Beauneveu et par Claus Sluter afin d'installer les figures dans des espaces fictifs profonds et vraisemblables. La présente contribution ambitionne d'explorer l'étape suivante de

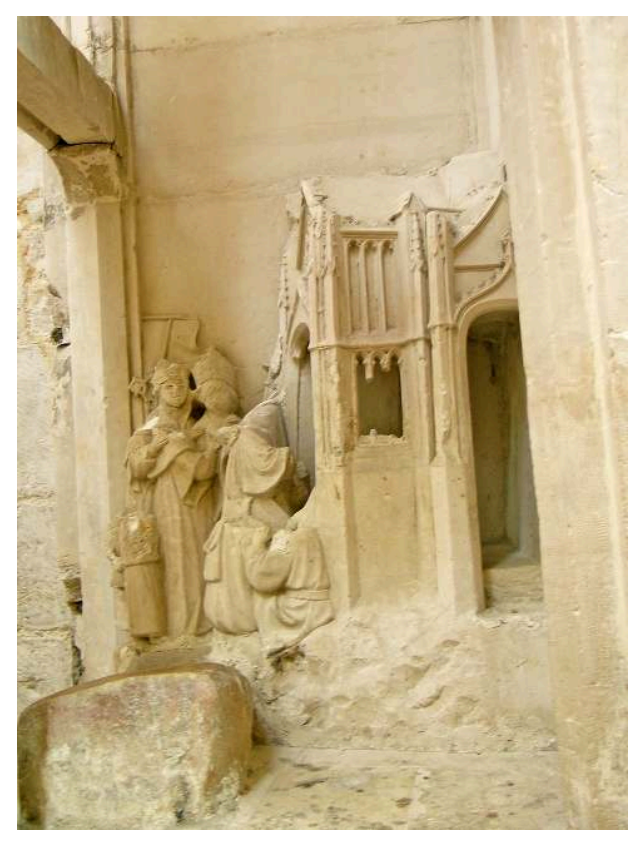
ces évolutions à partir de l'examen d'un édicule sculpté normand des années 1420 et de souligner ainsi les transformations radicales intervenues au début $\mathrm{du} \mathrm{xv}^{\mathrm{e}}$ siècle dans la figuration de l'espace de la représentation. 
Fig. 1 - Gauche : Champmol, tombeau de Philippe le Hardi, soubassement, 1385-1410 ; droite : Bourges, cathédrale Saint-Étienne, vitraux provenant de la Sainte-Chapelle, vers 1400

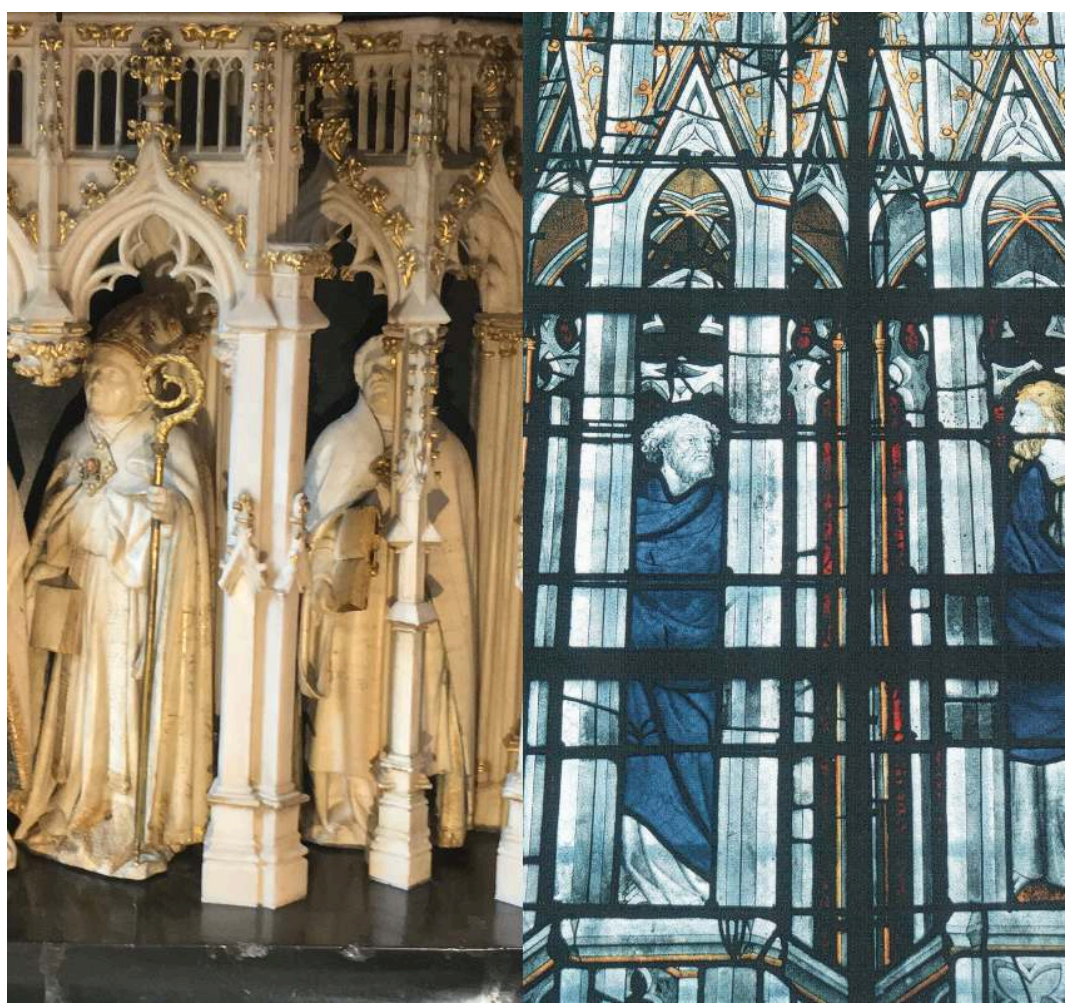

Gauche : cl. J.-M. Guillouët; droite : cl. F. Thomas

Le consensus est aujourd'hui établi pour attribuer la paternité des vitraux de la SainteChapelle de Bourges, déposés dans la crypte de la cathédrale, au grand sculpteur André Beauneveu (v. 1337-v. 1404), qui dut peut-être à sa place dans la familiarité de Jean de Berry d'avoir exercé ses talents dans des domaines divers, tels que la peinture sur manuscrit ou sur verre ${ }^{2}$. Dans ces vitraux, un soin particulier a ainsi été accordé à la manière dont les prophètes et les apôtres occupent des niches, dont les piles les dissimulent parfois dans un «jeu de cache-cache institué par ce cadre très présent 原 personnages, de face, de trois-quarts ou encore de plein profil ${ }^{3}$ ". Les édicules octogonaux ou carrés, dans lesquels les personnages sont enclos, sont présentés dans une perspective de sotto in sù. Les nervures de leurs voûtes retombent sur de hautes et massives colonnettes auxquelles s'adossent des contreforts de plan quadrangulaire. C'est derrière ces hautes colonnes que disparaissent, par intervalles, les figures pour conférer à l'espace du dais représenté une illusion de profondeur et une cohérence spatiale. La recherche de cette dernière, pour les espaces délimités par ces architectures de vitraux, est d'autant plus notable si l'on en compare les effets à ceux obtenus dans la verrière de Simon Aligret dans la cathédrale de Bourges, de peu postérieurs aux vitraux de la Sainte-Chapelle. Dans le domaine du vitrail, la fenêtre orientale de la chapelle Saint-Piat de la cathédrale de chartres est l'un des rares exemples contemporains ou antérieurs susceptibles d'être rapprochés des expériences de la Sainte-Chapelle berrichonne ${ }^{4}$. 
4 Pour retrouver une même conception innovante du cadre architectural, il faut quitter le domaine de la peinture sur verre pour se tourner vers la production sculptée et le tombeau de Philippe le Hardi, réalisé par Jean de Marville, Claus Sluter puis Claus de Werve pour l'église du couvent chartreux de Champmol. Dans cette œuvre exceptionnelle, s'observe le même renouvellement de la conception de l'espace représenté. La chronologie de ces travaux et la part de ses différents intervenants ont été l'objet de réévaluations récentes, qui ont permis de saisir l'ampleur des modifications introduites dans le projet de Jean de Marville par Claus Sluter, entre 1389 et 1406 , jusqu'à son achèvement par Claus de Werve en $1411^{5}$. Dans le très célèbre cortège des pleurants du soubassement se retrouvent les mêmes habiles dispositifs de composition et de construction spatiale employés dans les vitraux de Bourges. Les figures d'albâtre, aux physionomies et aux attitudes variées, ne sont pas seulement encadrées par l'arcature du soubassement, mais elles disparaissent occasionnellement derrière les piles recevant les retombées des voûtes. L'illusion de profondeur ainsi obtenue par cet " effet de cage ", dans lequel la figure apparait derrière les percées de son cadre architectural, contribue dès lors à construire cet «illusionnisme monumental » et à donner une impression de réalité à la scène.

5 Si la convergence de ces recherches peut être éclairée par la rencontre documentée des deux grands artistes à Mehun-sur-Yèvre en $1393^{6}$, ces inventions visuelles s'inscrivent surtout dans un mouvement plus général, touchant à la représentation de l'architecture et à la fonction alors conférée à cette dernière dans la construction de la cohérence topographique de la représentation. Le cadre monumental illusionniste est utilisé afin de suggérer la réalité d'un espace profond accueillant les figures. Ce constat est prolongé par des observations faites sur un petit ensemble sculpté, légèrement postérieur mais riche d'enseignement, le reliquaire dit du Pas de l'ange, situé aujourd'hui dans le bras sud de la célèbre abbatiale bénédictine de la Trinité de Fécamp (fig. 2) ${ }^{7}$. 
Fig. 2 - Fécamp, abbaye de la Trinité, Pas de l'ange, vue d'ensemble, 1420

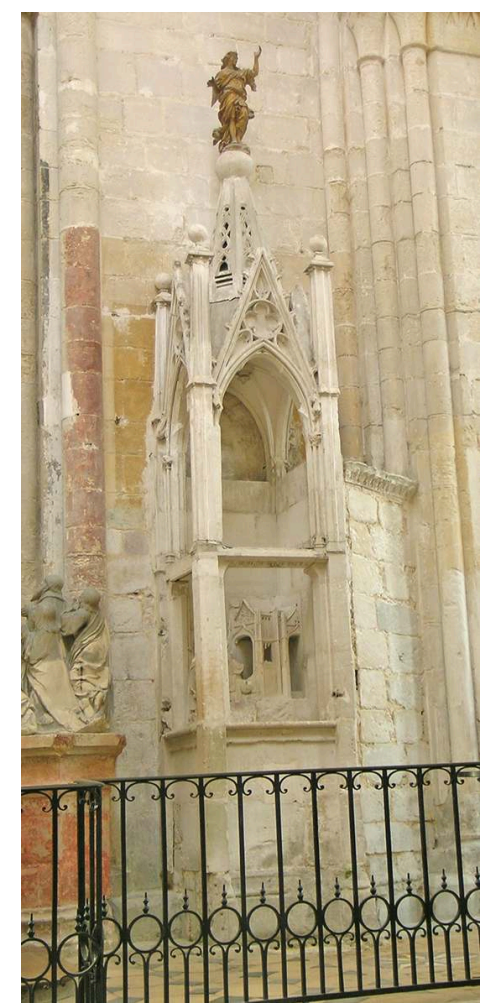

Cl. J.-M. Guillouët

6 Ainsi que le montre le contrat de la commande de cet édicule, passé en 1420 alors que les troubles politiques et militaires perduraient entre les royaumes anglais et français, les moines étaient alors désireux de rappeler la vénérable ancienneté de leur abbaye tout comme ses liens étroits avec les ducs de Normandie. Ils commandèrent pour cela la réalisation d'une scène sculptée rappelant la fondation légendaire de l'abbaye. La légende remonte en effet aux sources de l'institution qui avait été fondée en tant que monastère féminin par le seigneur franc Waningue, vers le milieu du viI ${ }^{e}$ siècle, et détruit par les Normands, deux siècles plus tard. À cet emplacement, vers 940, le duc normand Guillaume Longue-Épée (927-942) fonda et reconstruisit une chapelle. La légende raconte que, alors que le duc avait réuni les évêques normands pour décider de la dédicace de l'édifice, un pèlerin fit irruption et interrompit la discussion. Il entra dans l'édifice, déposa un couteau sur l'autel que l'on s'apprêtait à consacrer, puis s'envola miraculeusement en prenant appui sur une pierre dure qui conserva l'empreinte de son pied. Sur le couteau se lisait l'inscription: In Nomine Sanctae et Individuae Trinitatis. Ce miracle décida finalement de la dédicace du nouvel édifice qui prit le nom de la Trinité. La pierre, sur laquelle l'ange avait laissé l'empreinte de son pied, élevée au rang de relique indirecte, fut quelques décennies plus tard partagée en deux et, pour une moitié, ensevelie dans les fondations d'une nouvelle abbatiale construite à partir de juin 990 . Ce récit est bien connu et très largement repris dans toute la littérature sur le monument.

7 Lors de la commande d'un nouveau réceptacle pour cette relique insigne, la décision de représenter l'épisode miraculeux et légendaire s'imposait donc. Ce choix iconographique est mentionné dans le contrat passé en 1420 par les moines de l'abbaye avec Alexandre de Berneval, dont l'activité possède une certaine dimension 
entrepreneuriale. L'épisode fondateur n'est pas décrit précisément par les commanditaires, qui se contentent d'indiquer le thème de la sculpture - «l'ystore de la dedicasse »-, le nombre de personnages nécessaires - «jusquez au nombre de sept ymagez »-, leur dignité - «Evesquez, archevesquez, duc et pelerin»- ainsi que le cadre dans lequel doit se tenir la scène - "une apparence d'eglise ". La scène finalement sculptée comporte six figures principales et quatre figures annexes à la place des sept personnages initialement prévus (fig. 3).

Fig. 3 - Fécamp, abbaye de la Trinité, Pas de l'ange, détail de la scène sculptée, 1420

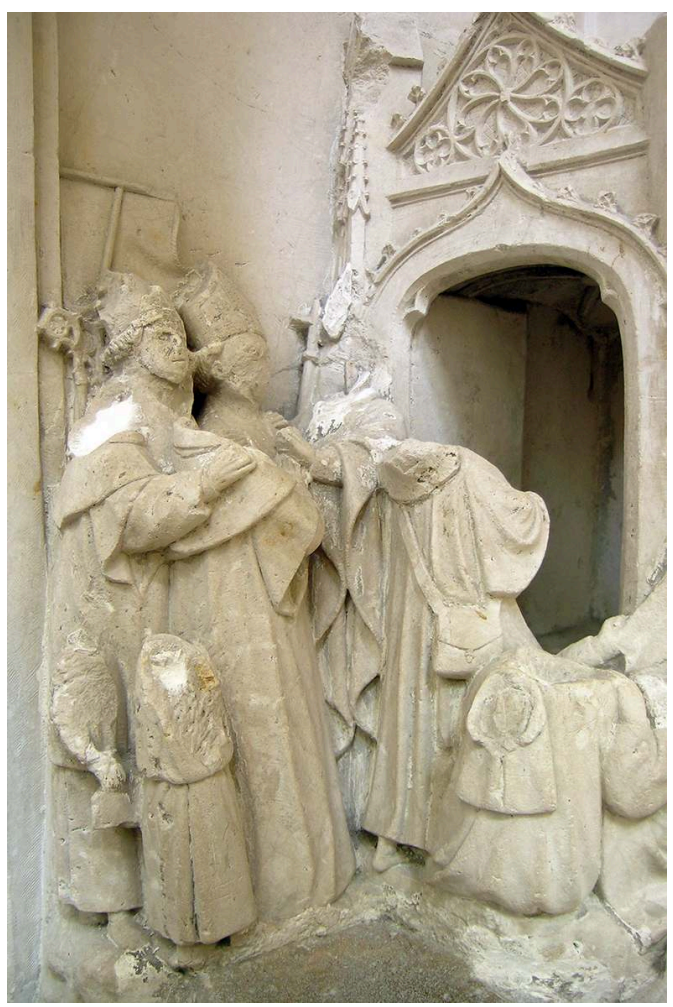

Cl. J.-M. Guillouët

Deux évêques mitrés, portant crosse, apparaissent dans le fond, sous une bannière flottante devant deux religieux en capuches figurant manifestement les moines de l'abbaye. Un personnage acéphale leur fait face et semble discuter avec eux dans l'angle du bâtiment miniature. On y reconnaît la figure mutilée du duc Guillaume Longue-Épée. Un dernier personnage principal (fragmentaire également), portant une bourse, pénètre dans l'église. Il s'agit de l'ange-pèlerin venu déposer le couteau sur l'autel à dédicacer.

Cette scène n'est pas la première, et moins encore l'unique représentation sculptée du miracle dans l'église abbatiale et c'est justement la comparaison avec la représentation lui ayant servi de modèle qui ouvre des perspectives intéressantes pour comprendre la logique de la microarchitecture médiévale. Ce même épisode légendaire apparaît en effet sur le long côté de la cuve du tombeau de l'abbé Thomas de Saint-Benoît (1297-1307), qui s'était fait inhumer dans le côté sud du chœur, alors nouvellement reconstruit, non loin de son prédécesseur Guillaume de Putot (1285-1297). Ce groupe de tombeaux ornés de scènes narratives et disposés dans le chœur a été l'objet d'une analyse de Georgia Sommers Wright, qui y reconnaissait un projet d'ensemble cohérent 
et le fruit de l'activité de deux ateliers, le premier actif à l'extrême fin du XIII siècle et le second dans les premières années du siècle suivant, dans la lignée du maître des statues d'Écouis .

Dans le groupe du tombeau (fig. 4), trois évêques mitrés, avec leur crosse, se tiennent debout dans le fond de la scène et font presque face à Guillaume Longue-Épée de manière assez semblable à ceux présents dans la sculpture du transept.

Fig. 4 - Fécamp, abbaye de la Trinité, tombeau de Thomas de Saint-Benoît, détail, début du $\mathrm{XIV}$ siècle

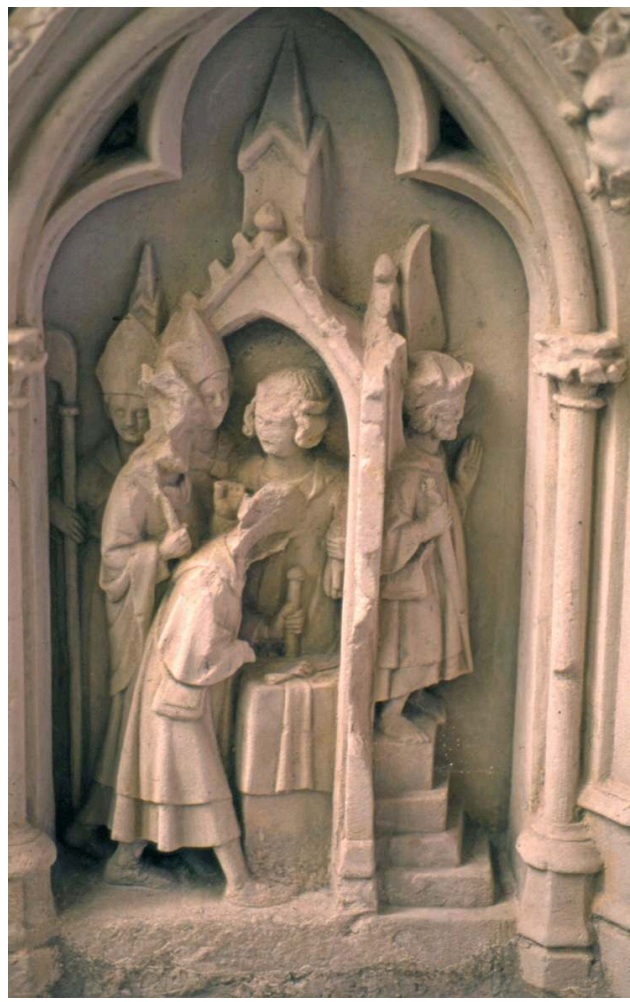

Cl. J.-M. Guillouët

11 Dans les deux cas, le pèlerin au premier plan, bourse pendant au côté, pénètre vers la droite dans l'édifice miniature ou s'approche, dans le premier ensemble, de l'autel du miracle. Dans la scène du XIV ${ }^{e}$ siècle cependant, le pèlerin apparaitt une seconde fois sur le pas d'une porte secondaire de l'édifice. Une aile, assez maladroitement figurée contre le fond de la scène, précise et complète le récit miraculeux. Dans l'édicule de 1420 (fig. 5), il n'apparaît qu'une seule fois, mais le flanc de la petite église est percé d'une porte similaire à l'entrée principale, d'où il est entendu que l'ange sortira pour s'envoler après avoir déposé le couteau sur l'autel. 
Fig. 5 - Fécamp, abbaye de la Trinité, Pas de l'ange, détail de la scène sculptée en vue latérale, 1420

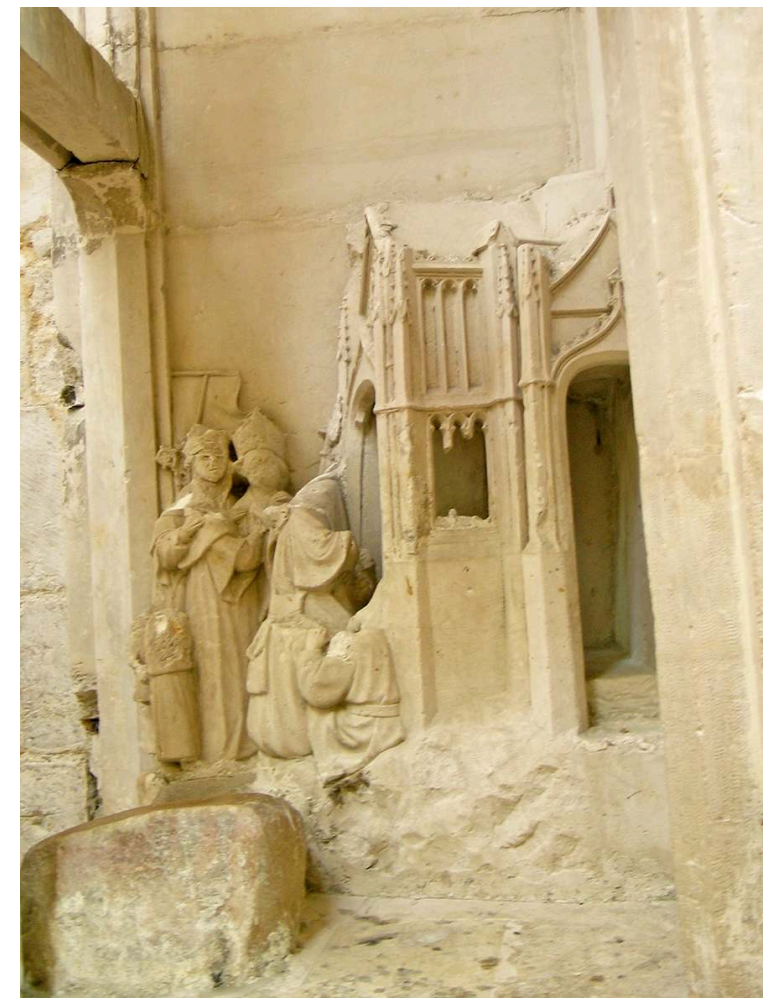

Cl. J.-M. Guillouët

12 Cette différence est fondamentale et oppose donc les deux scènes sculptées. Elle concerne le rôle assigné au décor architectural dans la narration ainsi que la nature de l'espace qu'il délimite. Dans la scène du tombeau de Thomas de Saint-Benoît, le pèlerin apparaît à deux reprises. Cette répétition construit une narration continue du miracle dans laquelle les épisodes successifs de la légende apparaissent simultanément dans la même scène : l'intrusion du pèlerin qui dépose le couteau sur l'autel et son envol sur le pas d'une porte secondaire de l'église 9 . Lew Andrew, à la suite de Dagobert Frey, reconnaît dans ce système un dispositif narratif proprement gothique. Il fait du Quattrocento italien une période de transition durant laquelle ces formes médiévales de la narration tendent à être remplacées par des dispositifs narratifs proprement modernes ${ }^{10}$.

L'exemple fécampois montre cependant que ces évolutions ne sont pas cantonnées à l'Italie, et cela, dès le début du Xve siècle. Dans le tombeau de Thomas de Saint-Benoît, cet aplanissement de la narration avait pour corollaire la mise en place d'un cadre architectural spatialement non cohérent. Le cadre architectural de la scène est constitué d'une arcade à cintre brisée surmontée d'un gâble-pignon, derrière lequel s'élève une tourelle coiffée d'une flèche. À droite, sont simplement adossées quatre marches, au sommet desquelles se tient l'ange-pèlerin dont les pieds nus sont ainsi bien mis en évidence. L'architecture est représentée de telle sorte que tous les protagonistes de la scène ainsi que l'autel du miracle se trouvent à la fois dans l'édifice miniature et en dehors de celui-ci. La grande arcade en tiers-point qui structure la composition possède ainsi d'abord une valeur indicielle. Elle est chargée de marquer l'emplacement de l'espace bâti dans lequel se déroule la scène, mais ne prétend pas délimiter un lieu ni l'espace d'une narration. 
14 Sur ce point, le groupe sculpté de 1420 diverge fortement de la scène du tombeau et témoigne d'une rupture radicale dans le rôle assigné au cadre architectural miniature pour la construction de l'espace de la narration. Il convient d'abord de souligner qu'aucun des deux volets principaux du miracle n'y apparaît: le pèlerin n'est pas montré déposant le couteau sur l'autel, pas plus qu'il n'est figuré prêt à s'élever dans les airs. À la place, le sculpteur a disposé le personnage entrant dans l'église ainsi que la porte secondaire par laquelle il est censé ressortir mais sur le seuil de laquelle il n'apparaît pas encore. L'ange-pèlerin n'est donc représenté qu'une seule et unique fois, alors qu'il interrompt la discussion du duc et des prélats pour pénétrer dans le bâtiment. Il incombe, dès lors, au spectateur de se figurer la suite de l'action devant se dérouler à l'intérieur de l'édifice miniature : dépose du couteau et envol miraculeux.

Cette disparition du miracle lui-même s'effectue au profit d'une cohérence topographique et narrative nouvelle par rapport à la scène du tombeau de Thomas de Saint-Benoît. Le fidèle n'est plus immédiatement et directement spectateur d'épisodes miraculeux, qui sont d'autant plus aisément suggérés qu'ils peuvent advenir dans un espace conçu désormais pour donner l'illusion d'un lieu parcourable. Le rôle premier de l'édifice miniature n'est plus d'ordre signalétique ou indiciel comme c'était le cas dans le tombeau de Thomas de Saint-Benoît. Les formes architecturales et leurs agencements ont ici pour rôle de délimiter un espace bâti vraisemblable et parcourable dans lequel l'action miraculeuse peut se dérouler. La microarchitecture acquiert de ce fait un statut nouveau, conditionné par cette parcourabilité nouvelle. À la manière des cabanes et/ou des ruines des images de la Nativité ou des Adorations étudiées par Silvia FabrizioCosta, l'édifice miniature du reliquaire du Pas de l'ange signale la volonté « de donner à voir une lisibilité optimale de l'histoire apte à susciter l'émotion dans la dévotion ${ }^{11}$ ». Si, dans le tombeau de Thomas de Saint-Benoit, l'architecture miniature a la même fonction que la cabane ou la ruine de la Nativité dans le récit biblique, en jouant le rôle d'un attribut permettant d'identifier l'épisode, elle sert surtout, dans le reliquaire de 1420, à circonscrire le lieu de l'action dans lequel se déploie la narration. Pour résumer plus encore et en constatant une nouvelle fois la disparition de tous les éléments du récit légendaire, on peut affirmer que l'objet de la représentation est dorénavant d'abord le lieu du miracle et non le miracle en lui-même.

La chronologie et la nature des observations précédentes, issues de la confrontation de ces deux scènes sculptées fécampoises séparées d'une centaine d'années, permettent de placer les jalons d'une nouvelle étape dans l'histoire des représentations de l'architecture à la fin du Moyen Âge ${ }^{12}$. C'est bien en effet à l'ourlet des $\mathrm{XIV}^{\mathrm{e}}$ et $\mathrm{XV}^{\mathrm{e}}$ siècles qu'il faut chercher les premières manifestations tangibles de cette transformation radicale intervenue dans la conception des figures du bâti. Du moins, pour les espaces septentrionaux du royaume et, plus spécifiquement, pour ceux de la France moyenne, un faisceau d'éléments convergents met en évidence l'importance des années 1400 dans la construction de nouveaux usages de la représentation de l'architecture et de cet illusionnisme monumental.

Plus précisément, les observations faites à partir du rapprochement des exemples précédents interrogent le rôle conféré au cadre architectural miniature de la narration - quand bien même celle-ci ne se compose que d'une figure isolée - dans la construction d'une cohérence spatiale nouvelle. La place centrale occupée dans ces évolutions par les décennies situées à l'articulation des $\mathrm{XIV}^{\mathrm{e}}$ et $\mathrm{XV}^{\mathrm{e}}$ siècles est encore confortée par d'autres exemples, comme celui des ébrasements du portail de la 
cathédrale Saint-Étienne d'Auxerre, daté des environs de $1400^{13}$. De récentes restaurations ont ainsi révélé un dispositif voisin de celui souligné dans le soubassement du tombeau de Champmol ou dans les vitraux de Bourges : dans la partie basse du portail d'Auxerre, devant les niches des ébrasements de ses portails, de fines colonnettes émergeaient de la banquette et s'élevaient pour rejoindre les retombées des voûtes couvrant les niches. Ces hautes et graciles colonnettes n'ont bien sûr pas résisté à l'érosion du temps et leur existence ancienne n'est plus attestée que par les traces de scellements retrouvées dans le lit d'attente des dernières assises du soubassement ${ }^{14}$. Les statues d'apôtres occupant ces niches se dessinaient cependant derrière ces hauts montants verticaux dont l'effet évoque aussitôt le parti que nous avons souligné à la suite de Fabienne Joubert. L'« effet de cage", produit par ces colonnettes masquant partiellement les figures de la niche, participe de ce même illusionnisme monumental visant à engager le spectateur dans l'élaboration intérieure d'un espace profond, cohérent et parcourable.

Cette convergence chronologique est notable et semble bien conduire à reconnaître, dans ces années entourant le tournant des $\mathrm{XIV}^{\mathrm{e}}$ et $\mathrm{XV}^{\mathrm{e}}$ siècles, un moment clef dans la transformation de la figuration de l'espace à travers le cadre de l'architecture miniature. Des dispositifs de composition sont en effet employés à Bourges, à Dijon ou à Auxerre vers 1400, avec ceci de commun qu'ils créent ou accentuent la profondeur en même temps qu'ils délimitent ou qu'ils circonscrivent des lieux dorénavant mentalement parcourables et habitables, là où précédemment l'encadrement architectural délimitait d'abord un cadre pour la figuration, servant à l'isoler.

Les transformations de l'espace représenté au Moyen Âge ainsi que le rôle de l'architecture dans ces évolutions soulèvent des questions complexes, touchant tout à la fois à l'histoire des représentations et à l'histoire intellectuelle et culturelle. Les usages médiévaux des termes de spatium et de locus n'obéissent bien sûr pas aux logiques contemporaines ${ }^{15}$. J.-C. Schmitt propose par ailleurs de reconnaitre dans les évolutions de la représentation - "unification des "lieux" de l'image, creusement du champ de la représentation sous le regard du spectateur, mise en séquence narrative, "naturalisation" partielle des thèmes "-, un écho de l'importance croissante de tous les processus de spatialisation dans les rapports sociaux au Moyen Âge central et tardif, phénomène mis en évidence par la recherche ces trente dernières années ${ }^{16}$. Nous retiendrons pour notre part que c'est la période du tournant des $\mathrm{XIV}^{\mathrm{e}}$ et $\mathrm{XV}^{\mathrm{e}}$ siècles qui est marquée par la constitution de cohérences topographiques nouvelles de l'image, à travers notamment les figures du bâti et l'architecture miniature.

De ce point de vue, le cas du reliquaire du Pas de l'ange de la Trinité de Fécamp, par lequel nous nous proposons de prolonger la réflexion engagée par Fabienne Joubert en 1999, parait illustrer très clairement ces évolutions. Dans la scène figurée du tombeau de Thomas de Saint-Benoît, les éléments architecturaux ont une fonction d'abord indicielle et non mimétique. L'édifice représenté est dénué de toute logique topographique. Ce sont les mêmes mécanismes qu'observe Jean-Claude Schmitt à propos de l'image du Saint Sépulcre dans l'enluminure de la Résurrection du Christ de l'Évangéliaire ottonien de Gniezno ${ }^{17}$. Dans l'édicule de 1420 , en revanche, si le bâtiment de l'arrière-plan n'est certes pas un portrait de l'abbatiale, il joue un rôle autre que purement signalétique. Sa présence est d'abord justifiée en ce qu'il délimite et circonscrit l'espace du récit qui s'impose dès lors comme le lieu du miracle. 
21 Il est demandé au pèlerin, agenouillé devant l'édicule pour prier la demi-pierre miraculeuse après avoir versé son obole dans le tronc - par la rainure que le contrat de 1420 appelle l'« heuze »-, de réaliser une opération de superposition mentale. Il s'agit d'abord pour lui de se figurer l'ange-pèlerin de la légende parcourir cet espace de la scène. Un espace que le fidèle ne voit pas, mais dont il perçoit la cohérence à travers le bâti miniature. C'est à l'intérieur de celui-ci qu'il doit imaginer les actions du miracle : la dépose du couteau sur l'autel puis l'envol de l'ange-pèlerin par la porte latérale de l'église. Dans le même temps et ce faisant, le pèlerin historique expérimente intérieurement un jeu d'équivalence ou, à tout le moins, de résonnance entre sa situation et celle de la légende, entre lui-même agenouillé devant la pierre du miracle et le pèlerin miraculeux de la geste sainte.

Cette "peregrinatio visionnaire ", c'est-à-dire cette déambulation intérieure n'est peutêtre pas sans rapport avec l'expérience réelle du pèlerinage ainsi que le suggère encore Jean-Claude Schmitt à propos du Liber Scivias d'Hildegarde de Bingen ${ }^{18}$. Il est en effet possible qu'une relation étroite, sinon même un lien de conséquence, ait existé entre la dispositio mentae du spectateur pérégrin et la construction de ces espaces parcourables où s'exerce la déambulation mentale de ce spectateur, cet «intellectual movement of the viewer " ainsi que le qualifie Lew Andrews ${ }^{19}$. À ce titre, l'édicule fécampois est un exemple particulièrement frappant de ces transformations du début $d u \mathrm{Xv}^{\mathrm{e}}$ siècle et vient en écho aux essais d'illusionnisme spatial rencontrés au même moment dans les cours des deux grands princes Valois du royaume, Jean de Berry et Philippe le Hardi. La convergence des expériences contemporaines d'André Beauneveu à Bourges, de Claus Sluter à Champmol ou des constructeurs du portail central de Saint-Étienne d'Auxerre nous paraît être le signe des évolutions plus générales touchant à la représentation de l'espace architectural et nous invite à faire des années 1400 un jalon majeur dans l'histoire de la conception de l'espace représenté et de l'usage de l'architecture miniature ${ }^{20}$.

Reçu : 9 janvier 2020 - Accepté : 7 avril 2020

\section{NOTES}

1. F. JOUBERT, "L'illusionnisme monumental à la fin du XIV siècle: les recherches d'André Beauneveu à Bourges et de Claus Sluter à Dijon ", in F. JOUBERT et D. SANDRON (dir.), Pierre, Lumière, couleur : études d'histoire de l'art du Moyen Âge en l'honneur d'Anne Prache, Paris, 1999, p. 367-384.

2. En dernier lieu et pour la bibliographie antérieure: S.NASH, «Les vitraux de la SainteChapelle ", in S. NASH (dir.), T. H. BORCHERT et T. HARRIS (coll.), "Sans égal en aucun pays " André Beauneveu. Artiste des cours de France et de Flandre, Londres, 2007, p. 154-177.

3. F. JOUBERT, «L'illusionnisme monumental... », op. cit., p. 370.

4. J. BUGSLAG, «Entre espace pictural et architectural. La fenêtre est de la chapelle Saint-Piat à la cathédrale de Chartres ", in Représentations architecturales dans les vitraux, Bruxelles, 2002 (Dossier de la Commission royale des monuments, sites et fouilles, 9), p. 85-94; p. 90-91.

5. Anciennement: H. DROUOT, «L'atelier de Dijon et l'exécution du tombeau de Philippe le Hardi. Notes critiques", Revue belge d'archéologie et d'histoire de l'art, 2/1 (janv. 1932), p. 11-39, ici p. 36 
(pièce 32) ; F. BARON, S. JUGIE et B. LAFAY, Les tombeaux des ducs de Bourgogne. Création, destruction, restauration, Paris, Somogy, 2009. Surtout, dernièrement, on consultera la complète réévaluation documentaire de Susie Nash ("The Two Tombs of Philip the Bold», Journal of the Warburg and Courtauld Institutes, 2019).

6. Les relations entre André Beauneveu et Claus Sluter ont été étudiées par Stephen K. Scher et, plus récemment, par Kathleen Morand: S. K. SCHER, « André Beauneveu and Claus Sluter », Gesta, 7 (1968), p. 3-14; K. MORAND, «Bourges et Dijon. Observations sur les relations entre André Beauneveu, Jean de Cambrai et Claus Sluter ", Actes des journées internationales Claus Sluter, Dijon, 1992, p. 277-288.

7. Reliquaire auquel j'avais consacré une étude en 2004 : J.-M. GUILLOUËT, « Une sculpture du $\mathrm{XV}^{\mathrm{e}}$ siècle et son contrat : le "Pas de l'ange" à la Trinité de Fécamp. De l'opportunité des légendes de fondation ", Bibliothèque de l'École des chartes, 162 (janvier-juin 2004), p. 133-161. Les éléments factuels des paragraphes qui suivent proviennent de ce dernier texte.

8. G.S. WRIGHT, "A tomb program at Fecamp», Zeitschrift für Kunstgeschichte, 47/2 (1984), p. 186-209.

9. Par opposition à une narration continue, telle qu'analysée par Lew Andrews pour la Renaissance: L. ANDREWS, Story and Space in Renaissance Art: the Rebirth of Continuous Narratives, Cambridge, 1995.

10. L. ANDREWS, Story and Space..., ibid., p. 6, citant également D. FREY, Gotik und Renaissance als Grundlagen der modernen Weltanschauung, Augsburg, 1929.

11. Dans le cas de ces thèmes, « le lieu de l'action doit permettre une grande visibilité de la scène (être dans, Nativité) et de l'action (aller vers, Adoration) », cf. s. FABRIzIO-cosTA, Entre trace(s) et signe(s) : quelques approches herméneutiques de la ruine, Berne, 2005. Il est intéressant de noter que les deux exemples que Silvia Fabrizio-Costa convoque dans sa démonstration, les deux Adoration des mages de Gentile da Fabriano et de Lorenzo Monaco conservées à la Galerie des Offices de Florence, sont précisément datés des mêmes années 1420 (ibid., p. 243). C'est aussi au début du $\mathrm{XV}^{\mathrm{e}}$ siècle que Lew Andrews situe une rupture importante dans la disparition de la narration continue médiévale et son remplacement par les modalités modernes de la narration, élaborée par des artistes en prise avec un réalisme plus prégnant (L. ANDREWS, Story and Space..., op. cit., p. 6). 12. Sur la microarchitecture et ses usages au Moyen Âge, je me permets de renvoyer à mon récent bilan historiographique: "Microarchitectures médiévales. Une perspective historiographique ", in J.-M. GUILLOUËT et A. VILAIN (dir.), Microarchitectures médiévales. L'échelle à l'épreuve de la matière, Paris, 2018, p. 25-34.

13. Et également étudié par Fabienne Joubert : F. JOUBERT, «Le portail central : la réalisation sous l'épiscopat de Michel de Creney, vers 1400, d'un programme ambitieux ", in C. SAPIN (dir.), SaintÉtienne d'Auxerre, la seconde vie d'une cathédrale. Sept ans de recherches pluridisciplinaires et internationales (2001-2007), Auxerre/Paris, 2011, p. 397-409. Le portail central de la cathédrale d'Auxerre présente en outre l'avantage d'être daté avec une grande fiabilité et une relative précision : les textes attestent que des travaux s'y déroulaient en 1397 et que les vantaux ont été posés en 1403.

14. H. HANSEN, "La chronologie relative des cinq portails", in C.SAPIN (dir.), Saint-Étienne d'Auxerre..., ibid., p. 220. S. AUMARD, « Le métal dans la construction de la cathédrale Saint-Étienne d'Auxerre ", in A. TIMBERT (dir.), L'homme et la matière. L'emploi du plomb et du fer dans l'architecture gothique, Paris, 2009, p. 159-172. F. JOUBERT, « Le portail central... », ibid., p. 403-406.

15. On consultera notamment : J.-C. sснмітт, « "De l'espace aux lieux" : les images médiévales », in Construction de l'espace au Moyen Âge: pratiques et représentations, Paris, 2007, p. 317-346 (surtout p. 320-324).

16. J.-C. schмiтt, « “De l'espace aux lieux”... », ibid., p. 345.

17. J.-C. schmitT, « "De l'espace aux lieux"... », ibid., p. 319. 
18. «L'espace [...] ne juxtapose pas des places et des coupes du Temple en accord avec les descriptions bibliques mais propose une peregrinatio visionnaire, qui crée ses propres lieux au fur et à mesure qu'elle progresse, et qui n'est peut-être pas sans rapport avec l'expérience du pèlerinage » (J.-C. schмiтT, « “De l'espace aux lieux”... », ibid., p. 331).

19. L. ANDREWS, Story and Space..., op. cit., p. 5.

20. Sur ces questions touchant aux procédures de la microarchitecture $\mathrm{du} \mathrm{xv}^{\mathrm{e}}$ siècle, voir J.M. GUILLOUËT, Flamboyant Architecture and medieval Technicality : the Rise of artistic Consciousness at the End of Middle Ages (ca 1400-ca 1530), Turnhout, 2019.

\section{AUTEUR}

\section{JEAN-MARIE GUILLOUËT}

Université de Bourgogne - Dijon, ARTEHIS (UMR 6298) 